Table S12. siRNAs sequences. Sequences of siRNAs (Qiagen) used in RNAi experiments of Gata4, Mef2a, Nkx2.5, and Srf in HL-1 cells.

\section{Product Name}

Gata4-si1

Gata4-si2

Mef2a-si1

Mef2a-si2

$\mathrm{Nkx2.5- \textrm {si } 1}$

$\mathrm{Nkx} 2.5$-si2

Srf-si1

Srf-si2

Gata4 1

Mm_Gata4_3

Mm_Mef2a 3

Mm_Mef2a 4

$\mathrm{Mm} \_\mathrm{Nkx} 2-\overline{5}$-3

Mm_Nkx2-5_4

Mm Srf 2

Mm_Srf_3

AllStars Negative Control

\section{Product ID}

SI01009799

SI01009813

SI01303449

SI01303456

SI01328257

SI01328264

SI00217133

SI00217140

siNon

1027280

Entrez ID

14463

14463

17258

17258

18091

18091

20807

20807

Non

Target MGI_Symbol

Gata4

Gata4

Mef2a

Mef2a

Nkx2-5

Nkx2-5

Srf

Sif

Synthetic

Sequence Accession Number

NM 008092

NM_008092

NM_001033713 NM_194070

NM 001033713 NM 194070

NM_008700

NM_008700

NM 020493

NM_020493

Synthetic
Sense Sequences

CTGGATTTAATTCGTATATAT

CACACAGAATAGCTTCATCAA

CACATTCTGCTGAATTATTTA

AAGTAATTATTAGGAATATAA

ACCCACGCCTTTCTCAGTCAA

CACGGGCACTTTCGACGGATT

CTCAATTTGCTATGAGTATTA

AGG GAC GGA ACC ACT TAT TTA

Unknown 